\title{
From the Continuity Problem of Set Potential to Georg Cantor Conjecture
}

\author{
Zhu Rongrong \\ DIEG Mathematics Research of HR, Fudan University, Shanghai, China
}

Email address:

rongrongzhu1969@163.com

\section{To cite this article:}

Zhu Rongrong. From the Continuity Problem of Set Potential to Georg Cantor Conjecture. American Journal of Applied Mathematics. Vol. 8, No. 4, 2020, pp. 216-222. doi: 10.11648/j.ajam.20200804.16

Received: May 8, 2020; Accepted: July 13, 2020; Published: July 28, 2020

\begin{abstract}
Background in 1878, Cantor puted forward his famous conjecture. Cantor's famous conjecture is whether there is continuity between the potential of the set of natural numbers and the potential of the set of real numbers. In 1900, Hilbert puted forward the first question of 23 famous mathematical problems at the International Congress of mathematicians in Paris. Purpose To study the continuity of set potential between the natural number set and the real number set, so as to provide mathematical support for the study of male gene fragment in human genome. Method The potential is extended by infinite division of sets and differential incremental equilibrium theory. There is a symmetry relation that the smallest element of infinite partition is 2 . When a set A corresponds to a subset of a set B one by one, but it can't make A correspond to B one by one, the potential of A is said to be smaller than that of $\mathrm{B}$. If $a$ is the potential of $\mathrm{A}$, and $b$ is the potential of $\mathrm{B}$, then $\mathrm{a}<\mathrm{b}$. We use $\sim \cdot 0$ to express the potential of natural number set and $\sim 1$ to express the potential of real number set. At present, it is not known whether there is a set $\mathrm{X}$, the potential of $\mathrm{X}$ satisfies $\sim \cdot 0<x<\sim \cdot 1$. Results There is no continuity problem in the set potential of the natural number set and the real number set, and four mixed potentials can be formed. It belongs to the category of super finite theory. Conclusion Cantor's conjecture is proved that potential of the natural number set and the real number set. That is, the potential of $\mathrm{X}$ satisfies $\sim 0<\mathrm{X}$ $<\sim 1$ does not exist.
\end{abstract}

Keywords: Natural Number Set, Real Number Set, Set Potential, Continuity Problem, Mixed Potential, Hyperfinite Theory, Infinite Classification

\section{Introduction}

\subsection{Cantor's Famous Conjecture Is Whether There Is Continuity Between the Potential of Natural Number Set and That of Real Number Set}

Further proof on the continuity of set potential. When a set A corresponds to a subset of a set B one by one, but it can't make A correspond to $\mathrm{B}$ one by one, the potential of $\mathrm{A}$ is said to be smaller than that of B. If $a$ is the potential of A, and $b$ is the potential of $\mathrm{B}$, then $\mathrm{a}<\mathrm{b}$. We use $\dot{\sim} 0$ to express the potential of natural number set and $\dot{\sim} 1$ to express the potential of real number set. At present, it is not known whether there is a set $\mathrm{X}$, the potential of $\mathrm{X}$ satisfies $\dot{\sim} 0$ $<x<\dot{\sim} 1$. Cantor put forward his famous conjecture that the above $\mathrm{X}$ set does not exist.

In order to study continuity of set potential between the natural number set and the real number set, four mixed potential are formed, which belong to the category of transfinite theory and are discontinuous set potential. The connection in the complexity of human genes is formed by the continuity of set potential. It is found that the complex pairing of genomes also has weak order and law, the continuity and controllability of the whole pairing potential of gene chain, and the discontinuity of DNA gene fragment, and the continuity of DNA forming chromosome skeleton to life body, so as to ensure the relative stability of species.

\subsection{The continuity Problem of Proving Set Potential in Detail}

Sets $A^{i} \subset A, B^{i} \subset B$, potential $a^{i} \leftrightarrow b^{i} \subset A$, and $a^{i} \leftrightarrow$ $b^{i} \subset B$

$\forall a^{i} \leftrightarrow b^{i} \subset A, \forall a^{i} \leftrightarrow b^{i} \nsubseteq B$

Let $a^{i}$ is $\dot{\sim}^{i} 0, b^{i}$ is $\dot{\sim}^{i} 1$,

If $\forall a^{i} \leftrightarrow b^{i} \subset A$ 


$$
\begin{aligned}
& \forall \dot{\sim} 0 \leftrightarrow \dot{\sim} 1 \subset \dot{\sim}^{i} 0, \forall \dot{\sim} 0 \leftrightarrow \dot{\sim} 1 \not \subset \dot{\sim}^{i} 1 \\
& \forall \dot{\sim} 0 \leftrightarrow \dot{\sim} 1 \not \subset \dot{\sim}^{i} 0, \forall \dot{\sim} 0 \leftrightarrow \dot{\sim} 1 \subset \dot{\sim}^{i} 1
\end{aligned}
$$

The potential of the set of $\exists X$ is $X^{i}$

$$
X^{i} \subset \dot{\sim} 1, \dot{\sim} 0<x<\dot{\sim} 1 X^{i} \subset \dot{\sim}^{i} 0, \nexists \dot{\sim} 0<x<\dot{\sim} 1
$$

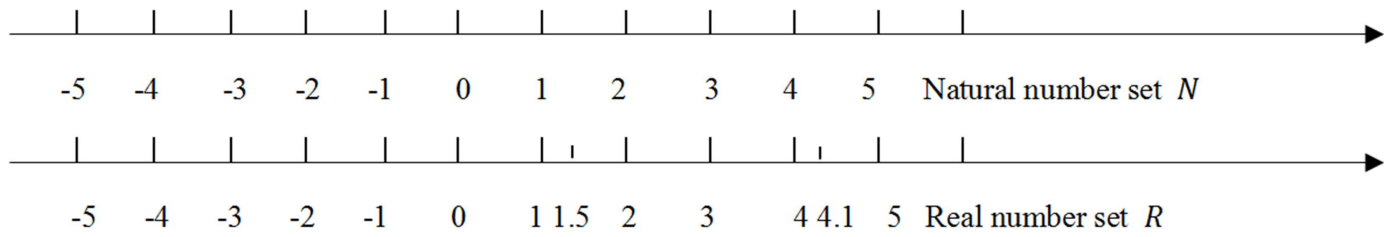

Figure 1. Coordinate axes of natural number set and real number set [set potential].

Subsets of set $N$ of natural numbers $a=\{0,1,2,3,4,5\}, a \subset N$

Subsets of real set R set $b=\{0,1,1.5,2,3,4,4.1,5\}, b \subset R$

Sets $c=\{0,1,2,3,4,5\}, c \subset N \subset R$

$a, b, c$ All known as, A is the potential of $\mathrm{N}$ set, $\mathrm{B}$ is the potential of $\mathrm{R}$ set, $\mathrm{C}$ is the potential of $\mathrm{N}$ set.

$\forall a, c$, potential $a<b \not \subset \mathrm{a}<x^{i}<b$

$\forall a, c$, One to one corresponding subset, and a, $\mathrm{c}$ are natural numbers.

Potential $a<b \not \subset \mathrm{a}<x^{i}<b, \sim 0 \nless x<\dot{\sim} 1$

$$
\begin{aligned}
& \dot{\sim} 0<x \nless \dot{\sim} 1 \\
& \dot{\sim} 0 \nless x \nless \dot{\sim} 1
\end{aligned}
$$

From the above not strict derivation, it may be considered that $\dot{\sim} 0<x<\dot{\sim} 1$ does not exist A strict, systematic and structured deep mathematical proof of $\dot{\sim} 0<x<\dot{\sim} 1$

From $a=\{0,1,2,3,4,5\}, b=\{0,1,1.5,2,3,4,4.1,5\}$, there is

$$
a=\{0,1,2, \ldots, n\}, b=\{0,1,2, \ldots, R\}
$$

Potential $\forall \bigcup_{i=1}^{m} a^{0^{-}}, i=1,2, \ldots, m, m+1, \ldots, n$; potential $\forall \bigcup_{j=1}^{p} b^{0^{-}}, j=1,2, \ldots p, p+1, \ldots, n$

Subset $\forall \cup_{i=1}^{m} \Delta a^{0^{-}} \subset \forall(\cup)\left[\int^{\Delta} b^{0^{-}}+\Delta a^{0^{-}}\right], \Delta a^{0^{-}}<\int^{\Delta} b_{i}^{0^{-}}$, Analysis from set unit

$$
\forall \bigcup_{i=1}^{m} \Delta a^{0^{-}}=\left\{\Delta a_{1}^{0^{-}}, \Delta a_{2}^{0^{-}}, \Delta a_{3}^{0^{-}}, \ldots, \Delta a_{k}^{0^{-}}, \ldots\right\}, \forall(\mathrm{U})\left[\int^{\Delta} b^{0^{-}}+\Delta a^{0^{-}}\right]=\left[\int^{\Delta} b_{1}^{0^{-}}, \Delta a_{2}^{0^{-}}, \int^{\Delta} b_{3}^{0^{-}}, \ldots, \Delta a_{k+1}^{0^{-}}, \Delta a_{k}^{0^{-}}, \ldots\right]
$$

Explain $\Delta a_{2}^{0^{-}}=\Delta^{*} a_{2}^{0^{-}}, \Delta a_{k}^{0^{-}}=\Delta^{*} a_{k+1}^{0^{-}}+\int^{\Delta} b_{1}^{0^{-}}$

$\forall(\mathrm{U}) \Delta a^{0^{-}}$takes a subset, i.e. potential a, so that $a=\dot{\sim} 0$

Take a subset from $\forall(\mathrm{U})\left[\int^{\Delta} b^{0^{-}}+\Delta a^{0^{-}}\right]$, potential b

Let $b=\dot{\sim} 1$

When $\forall(\mathrm{U}) \Delta a^{0^{-}}$and $\forall(\mathrm{U})\left[\int^{\Delta} b^{0^{-}}+\Delta a^{0^{-}}\right]$is infinitely classification, there is $\forall(\mathrm{U}) \Delta a^{0^{-}} \approx \forall(\mathrm{U})\left[\Delta \mathrm{a}^{0^{--}}+\operatorname{Lim}^{\Delta} 0^{\infty} b^{0^{-}}\right]$

Further structure

$$
\forall(\mathrm{U}) \Delta a_{i}^{0^{-}} \approx \forall(\mathrm{U})\left[\Delta a_{j}^{0^{i-}}+\operatorname{Lim} \int^{\Delta} 0^{\infty} b_{j}^{0^{-}}\right]
$$

From formula (2), we can see that there is no comparability of set $\mathrm{X}$, so

$$
\text { Let } \forall(\mathrm{U}) \Delta a_{i}^{0^{-}} \rightarrow a^{*}, \forall(\mathrm{U})\left[\Delta a_{j}^{0^{i-}}-\operatorname{Lim} \int^{\Delta} 0^{-} b_{j}^{0^{-}}\right] \rightarrow b^{*}
$$

Let $a^{*} \rightarrow \dot{\sim} 0, b^{*} \rightarrow \dot{\sim} 1$ again, and suppose that there is an $\mathrm{X}$ set, then there is $\dot{\sim} 0 \approx x \approx \dot{\sim} 1$

Therefore, $\dot{\sim} 0 \nless x \nless \dot{\sim} 1$

$$
\forall(\mathrm{U}) \Delta a_{i}^{0^{-}} \approx \forall(\mathrm{U})\left[\Delta a_{j}^{0^{i-}}-\operatorname{Lim} \int^{\Delta} 0^{\infty} b_{j}^{0^{-}}\right]
$$

Further analyze (2) and sort out the formula. 


$$
\begin{gathered}
\forall(\mathrm{U}) \Delta a^{0^{-}}, \forall(\mathrm{U})\left[\Delta a^{0^{-}}+\int^{\Delta} b^{0^{-}}\right] \\
\forall(\mathrm{U}) \Delta a^{0^{-}} \approx \forall(\mathrm{U})\left[\Delta \mathrm{a}^{0^{--}}+\operatorname{Lim} \int^{\Delta} 0^{\infty} b^{0^{-}}\right] \\
\forall(\mathrm{U}) \Delta a_{i}^{0^{-}} \approx \forall(\mathrm{U})\left[\Delta a_{j}^{0^{i-}}+\operatorname{Lim} \int^{\Delta} 0^{\infty} b_{j}^{0^{-}}\right] \\
\forall(\mathrm{U}) \Delta a_{i}^{0^{-}} \rightarrow a^{*} \rightarrow \dot{\sim} 0, \forall(\mathrm{U})\left[\Delta a_{j}^{0^{i-}}+\operatorname{Lim} \int^{\Delta}{ }^{\infty}{ }^{-} b_{j}^{0^{-}}\right] \rightarrow b^{*} \rightarrow \dot{\sim} 1 \\
\forall(\mathrm{U}) \Delta a_{i}^{0^{-}} \subset \forall(\mathrm{U})\left[\Delta a_{j}^{0^{i-}}+\operatorname{Lim} \int^{\Delta}{ }^{\infty} b_{j}^{0^{-}}\right]
\end{gathered}
$$

For (2) and (8), take a set $\mathrm{X}$

$$
\begin{gathered}
\forall(\mathrm{U})\left[\Delta a_{j}^{0^{i-}}+\operatorname{Lim} \int^{\Delta} 0^{-} b_{j}^{0^{-}}\right] \rightarrow \forall(\mathrm{U}) \Delta a_{j}^{0^{i-}} \\
\forall(\mathrm{U}) \Delta a_{j}^{0^{i-}}=\left\{\forall(\mathrm{U}) \Delta a_{i+\varepsilon}^{0^{i-}}+\forall(\mathrm{U}) \Delta a_{i+\varepsilon+1}^{0^{i-}}+\cdots+\forall(\mathrm{U}) \Delta a_{i+\varepsilon+\eta}^{0^{i-}}\right\}
\end{gathered}
$$

For $\varepsilon=0,(10)$ becomes

$$
\forall(\mathrm{U}) \Delta a_{j}^{0^{i-}}=\left\{\forall(\mathrm{U}) \Delta a_{i}^{0^{-}}+\forall(\mathrm{U}) \Delta a_{i+1}^{0^{--}}+\cdots+\forall(\mathrm{U}) \Delta a_{i+\eta}^{0^{i-}}\right\}
$$

(11) Where, $\forall(U) \Delta a_{i}^{0^{-}}$is the same as (2) or (5); the potential $\forall(U) \Delta a_{i}^{0^{-}}$at the left end is the same, so as to distinguish them (they belong to different set potentials),

So, change the $\forall(U) \Delta a_{i}^{0^{-}}$in formula (11) to $\forall(U) \Delta^{*} a_{i}^{0^{-}}$

$$
\forall(\mathrm{U}) \Delta a_{i}^{0^{-}} \stackrel{?}{\sim} \forall(\mathrm{U}) \Delta^{*} a_{i}^{0^{-}}
$$

The real number set $\mathrm{R}$ can be written as

$$
\forall(U)\left[\Delta a_{j}^{0^{i-}}+\operatorname{Lim} \int^{\Delta} 0^{-} b_{j}^{0^{-}}\right]
$$

from which it can be separated:

$$
\forall(\mathrm{U}) \Delta a_{j}^{0^{i-}}=\left\{\forall(\mathrm{U}) \Delta a_{i}^{0^{-}}+\forall(\mathrm{U}) \Delta a_{i+1}^{0^{--}}+\cdots+\forall(\mathrm{U}) \Delta a_{i+\eta}^{0^{i-}}\right\}, \text { and } \forall(\mathrm{U}) \Delta a_{i}^{0^{-}} \stackrel{?}{\sim} \forall(\mathrm{U}) \Delta^{*} a_{i}^{0^{-}}
$$

$\Delta^{*} a_{i}^{0^{-}} \not \subset N, \Delta^{*} a_{i}^{0^{-}} \subset R, R$ real number;

$\forall(U) \Delta a_{i}^{0^{-}} \subset N, N$ Natural number

Because $\mathrm{N}$ is a natural number and has continuity, $\forall(U) \Delta a_{i}^{0^{-}}$is also continuous; however, $\forall(U) \Delta^{*} a_{i}^{0^{-}}$is continuous.

$$
\therefore \Delta^{*} a_{i}^{0^{-}} \not \subset N, \Delta^{*} a_{i}^{0^{-}} \subset R
$$

Subset of set of natural numbers, Subset a of potential $\forall(U) \Delta a_{i}^{0^{-}}$, potential $a$. Subset of set of real numbers, Subsets $b$ of potential $\Delta^{*} a_{i}^{0^{-}}$, potential $b$.

Let $a^{*} \rightarrow \dot{\sim} 0, b^{*} \rightarrow \dot{\sim} 1$ The potential of $a$ and $b$ are respectively:

$$
\begin{aligned}
& a \subset \forall(\cup) \Delta a_{i}^{0^{-}}, a \not \subset \Delta^{*} a_{i}^{0^{-}} \\
& b \not \subset \forall(\cup) \Delta a_{i}^{0^{-}}, b \subset \Delta^{*} a_{i}^{0^{-}}
\end{aligned}
$$

$\therefore b$ continuous or not

Assume $\forall x \subset X$, then $\forall X \subset \forall(\mathrm{U}) \Delta a_{i}^{0^{-}}, \forall x \subset \forall(\mathrm{U}) \Delta^{*} a_{i}^{0^{-}}$

$\forall(\mathrm{U}) \Delta a_{i}^{0^{-}} \sim \forall(\mathrm{U}) \Delta^{*} a_{i}^{0^{-}}$equivalent or not, and

$\forall(\mathrm{U}) \Delta a_{i}^{0^{-}}, \forall(\mathrm{U}) \Delta^{*} a_{i}^{0^{-}} \subset N$,

Whether it is established, and Whether $\dot{\sim} 0<x<\dot{\sim} 1$

is established

For formula (7), detailed analysis

$$
\forall(\mathrm{U})\left[\Delta a_{j}^{0^{i-}}+\operatorname{Lim} \int^{\Delta}{ }^{\infty} b_{j}^{0^{-}}\right] \rightarrow \forall(\mathrm{U}) \Delta a_{j}^{0^{i-}}
$$




$$
\forall(\mathrm{U}) \Delta a_{j}^{0^{i-}}=\left\{\forall(\mathrm{U}) \Delta a_{i+\varepsilon}^{0^{--}}+\forall(\mathrm{U}) \Delta a_{i+\varepsilon+1}^{0^{--}}+\cdots+\forall(\mathrm{U}) \Delta a_{i+\varepsilon+\eta}^{0^{i-}}\right\}
$$

Because the value of $\varepsilon, \eta$ is continuous, that is, $\varepsilon=1,2, \ldots, n ; \eta=1,2, \ldots, m$

$$
\begin{aligned}
& \forall(\mathrm{U} \quad) \Delta a_{i}^{0^{-}} \approx \forall(\mathrm{U})\left[\Delta a_{j}^{0^{i-}}+\operatorname{Lim} \int^{\Delta}{ }_{0^{-}}^{\infty} b_{j}^{0^{-}}\right], \text {andi, } j, \varepsilon=1,2, \ldots \\
& \forall(U)\left[\Delta a_{j}^{0^{i-}}+\operatorname{Lim} \int^{\Delta} 0^{\infty} b_{j}^{0^{-}}\right] \\
& =\left\{\forall(U) \Delta a_{i+1}^{0^{--}}+\operatorname{Lim} \int^{\Delta} 0^{\infty} b_{\varepsilon}^{0^{i-}}+\forall(U) \Delta a_{i}^{0^{---}}+\operatorname{Lim} \int^{\Delta}{ }_{0^{-}}^{\infty} b_{\varepsilon+1}^{0^{(i+1)-}}, \ldots, \forall(U) \Delta a_{i}^{0^{i-}}+\operatorname{Lim} \int^{\Delta}{ }^{\infty} b_{\varepsilon+\eta}^{0^{(i+\eta)-}}, \ldots\right\} \\
& \forall(\mathrm{U}) \Delta a_{i+1}^{0^{-}} \approx\left\{\forall(\mathrm{U}) \Delta a_{i+1}^{0^{--}}+\forall(\mathrm{U}) \Delta a_{i}^{0^{---}}+\cdots+\forall(\mathrm{U}) \Delta a_{i}^{0^{i-}}\right\} \\
& \operatorname{Lim} \int^{\Delta}{ }_{0^{-}}^{\infty} b_{j+\varepsilon}^{0^{-}} \approx \operatorname{Lim} \int^{\Delta}{ }^{\infty} b_{\varepsilon}^{0^{i-}}+\operatorname{Lim} \int^{\Delta} 0^{\infty} b_{\varepsilon+1}^{0^{(i+1)-}}+\cdots+\operatorname{Lim} \int^{\Delta}{ }_{0}^{\infty} b_{\varepsilon+\eta}^{0^{(i+\eta)-}} \\
& \forall(\mathrm{U}) \Delta a_{i-1}^{0^{-}} \approx\left\{\forall(\mathrm{U}) \Delta a_{i-1}^{0^{--}}+\forall(\mathrm{U}) \Delta a_{i}^{0^{---}}+\cdots+\forall(\mathrm{U}) \Delta a_{i-1}^{0^{(i-1)^{-}}}\right\} \\
& \forall(\mathrm{U}) \Delta a_{i}^{0^{-}} \approx\left\{\forall(\mathrm{U}) \Delta a_{i}^{0^{--}}+\forall(\mathrm{U}) \Delta a_{i}^{0^{---}}+\cdots+\forall(\mathrm{U}) \Delta a_{i}^{0^{i^{-}}}\right\}
\end{aligned}
$$

So, Subset of set of natural numbers, potential $\forall(U) \Delta a_{i}^{0^{-}}$, and subset of set of real numbers, potential

$$
\forall(\mathrm{U})\left[\Delta a_{i-1}^{0^{-}}+\operatorname{Lim} \int^{\Delta}{ }^{\infty}{ }^{-} b_{j+\varepsilon}^{0^{-}}\right] \approx \forall(\mathrm{U}) \Delta^{*} a_{i}^{0^{-}}, \therefore \forall(\mathrm{U}) \Delta a_{i}^{0^{-}} \neq \forall(\mathrm{U}) \Delta^{*} a_{i}^{0^{-}}
$$

If $\forall(\mathrm{U}) \Delta a_{i}^{0^{-}} \equiv \forall(\mathrm{U}) \Delta^{*} a_{i}^{0^{-}}$, then $\dot{\sim} 0<x<\dot{\sim} 1$ established.

If $\forall(\mathrm{U}) \Delta a_{i}^{0^{-}} \neq \forall(\mathrm{U}) \Delta^{*} a_{i}^{0^{-}}$, then $\dot{\sim} 0<x<\dot{\sim} 1$ not established.

$\forall(U)\left[\Delta a_{i-1}^{0^{-}}+\operatorname{Lim} \int^{\Delta}{ }_{0^{-}}^{\infty} b_{j+\varepsilon}^{0^{-}}\right]$is a discontinuous potential, and $\forall(U) \Delta^{*} a_{i}^{0^{-}}$is a discontinuous potential, that is the potential of R (set of real numbers).

$\forall(\mathrm{U}) \Delta a_{i}^{0^{-}}$is a continuous potential, that is, the potential of $\mathrm{N}$ (set of natural numbers).

$\because \forall(\mathrm{U}) \Delta a_{i}^{0^{-}}$and $\forall(\mathrm{U}) \Delta^{*} a_{i}^{0^{-}}$are the potentials of the minimum set. $\forall x \in \forall(\mathrm{U}) \Delta a_{i}^{0^{-}}, \forall x \in \forall(\mathrm{U}) \Delta^{*} a_{i}^{0^{-}}$

$\because \forall_{\text {Min }}(\mathrm{u}) \Delta a_{i}^{0^{-}} \neq \forall_{\text {Min }}(\mathrm{u}) \Delta^{*} a_{i}^{0^{-}}$changed to $\forall_{0}^{-\infty}(\mathrm{u}) \Delta a_{i}^{0^{-}} \neq \forall_{0}^{-\infty}(\mathrm{u}) \Delta^{*} a_{i}^{0^{-}}$,

$\therefore \dot{\sim} 0<x<\dot{\sim} 1$ not established.

$$
\begin{aligned}
& \forall(\mathrm{U})\left[\Delta a_{i-1}^{0^{-}}+\operatorname{Lim} \int^{\Delta} 0^{-} b_{j+\varepsilon}^{0^{-}}\right] \approx \forall(\mathrm{U}) \Delta^{*} a_{i}^{0^{-}}, \forall_{0}^{-\infty}(\mathrm{U}) \Delta a_{i}^{0^{-}} \neq \forall_{0}^{-\infty}(\mathrm{U}) \Delta^{*} a_{i}^{0^{-}}, \\
& \therefore \dot{\sim} 0<x<\dot{\sim} 1 \text { not established }
\end{aligned}
$$

Explain: $\operatorname{Lim} \int^{\Delta}{ }^{\infty}{ }^{-} b_{j+\varepsilon}^{0^{-}}$in $\forall(\mathrm{U})\left[\Delta a_{i-1}^{0^{-}}+\operatorname{Lim} \int^{\Delta}{ }^{\infty}{ }^{-} b_{j+\varepsilon}^{0^{-}}\right]$is always with $\forall(\mathrm{U}) \Delta a_{i-1}^{0^{-}}$

About $\forall(U)\left[\Delta a_{i-1}^{0^{-}}+\operatorname{Lim} \int^{\Delta}{ }^{\infty} b_{j+\varepsilon}^{0^{-}}\right]$

$$
\begin{gathered}
\forall(\mathrm{U}) \Delta a_{i-1}^{0^{-}} \approx \forall(\mathrm{U}) \Delta a_{i-1}^{0^{--}}+\forall(\mathrm{U}) \Delta a_{i-1}^{0^{---}}+\cdots+\forall(\mathrm{U}) \Delta a_{i-1}^{0^{(i-1)^{-}}} \\
\operatorname{Lim} \int^{\Delta}{ }_{0^{\infty}}^{\infty} b_{j+\varepsilon}^{0^{-}}=\operatorname{Lim} \int^{\Delta}{ }_{0^{-}}^{\infty} b_{\varepsilon}^{0^{-}}+\operatorname{Lim} \int^{\Delta}{ }_{0^{-}}^{\infty} b_{\varepsilon+1}^{0^{(i-1)^{-}}}+\cdots+\operatorname{Lim} \int^{\Delta}{ }_{0^{-}}^{\infty} b_{\varepsilon+\eta}^{0^{(i+\eta)^{-}}}
\end{gathered}
$$

For (20), take the limit

$$
\operatorname{Lim}\left[\forall(\mathrm{U}) \Delta a_{i-1}^{0^{-}}\right] \approx \operatorname{Lim}\left[\forall(\mathrm{U}) \Delta a_{i-1}^{0^{--}}+\forall(\mathrm{U}) \Delta a_{i-1}^{0^{---}}+\cdots+\forall(\mathrm{U}) \Delta a_{i-1}^{0^{(i-1)^{-}}}\right]
$$


Discuss the relationship between $\forall(\mathrm{U}) \Delta a_{i}^{0^{-}}$and $\forall(\mathrm{U}) \Delta^{*} a_{i}^{0^{-}}$

$\forall(U)\left[\Delta^{*} a_{i-1}^{0^{-}}+\operatorname{Lim} \int^{\Delta}{ }^{\infty} b_{j+\varepsilon}^{0^{-}}\right] \leftrightarrow \forall(U) \Delta a_{i}^{0^{-}}, \because \operatorname{Lim} \int^{\Delta}{ }^{\infty} b_{j+\varepsilon}^{0^{-}}$is not potential ofset of natural numbers.

$\operatorname{Lim} \int^{\Delta}{ }^{\infty}{ }^{-} b_{j+\varepsilon}^{0^{-}} \operatorname{in} \forall(\mathrm{U})\left[\Delta^{*} a_{i-1}^{0^{-}}+\operatorname{Lim} \int^{\Delta}{ }^{\infty}{ }^{-} b_{j+\varepsilon}^{0^{-}}\right]$is always with $\Delta^{*} a_{i-1}^{0^{-}}$

$\operatorname{Lim} \int^{\Delta}{ }^{\infty} b_{j+\varepsilon}^{0^{-}}$is the infinitesimal of infinitesimal; it is the category of hyperfinity.

$\forall(\mathrm{U}) \Delta a_{i}^{0^{-}} \neq \forall(\mathrm{U})\left[\Delta^{*} a_{i-1}^{0^{-}}+\operatorname{Lim} \int^{\Delta}{ }_{0^{-}}^{\infty} b_{j+\varepsilon}^{0^{-}}\right]$, and In infinitesimal, infinitesimal set potential, It is always accompanied by $\operatorname{Lim} \int^{\Delta} 0^{-} b_{j+\varepsilon}^{0^{-}}$

$\therefore \dot{\sim} 0<x<\dot{\sim} 1$ not established

That is, the potential element of the set of $2, \dot{\sim} 0 \in N$ natural numbers set in $\dot{\sim} 0$; and the unit of the potential of $2, \dot{\sim} 1 \in R$ real number set in $\dot{\sim} 1$.

$\dot{\sim} 0 \rightarrow 2 \neq \dot{\sim} 1 \rightarrow 2$, that is $2 \neq 2^{*}$. It shows that the set potential has a great influence on the meaning of numbers.

\section{This Paper Discusses the Further Understanding of $\forall(U) \Delta \boldsymbol{a}_{\boldsymbol{i}}^{\mathbf{0}^{-}}$and $\forall(U) \Delta^{*} \boldsymbol{a}_{\boldsymbol{i}}^{\mathbf{0}^{-}}$to the from the Meaning of Infinite Classification of Sets}

2.1. The Meaning of Infinite Classification, the Smallest Element is 2, i.e. $2\{1 \rightarrow 1\}$, One-to-One Correspondence

$$
\begin{gathered}
\forall\{1 \rightarrow 1\} \in 2\{1 \rightarrow 1\} ; \forall(1), \forall_{\rightarrow}(1) \in 2\{1 \rightarrow 1\} \\
\left\{\begin{array}{c}
\forall(1), \forall(U) \Delta a_{i}^{0^{-}} \because \forall(1), \forall_{\rightarrow}(1) \in 2\{1 \rightarrow 1\} \\
\forall_{\rightarrow}(1), \forall(U) \Delta^{*} a_{i}^{0^{-}} \therefore 2\left\{\forall(1), \forall_{\rightarrow}(1)\right\}=2\{1 \rightarrow 1\}
\end{array}\right.
\end{gathered}
$$

From $2\left\{\forall(1), \forall_{\rightarrow}(1)\right\}=2\{1 \rightarrow 1\}$, it can be deduced.

$$
2\left\{\forall(\mathrm{U}) \Delta a_{i}^{0^{-}}, \forall(\mathrm{U}) \Delta^{*} a_{i}^{0^{-}}\right\}=2\left\{\forall(\mathrm{U}) \Delta a_{i}^{0^{-}} \rightarrow \forall(\mathrm{U}) \Delta^{*} a_{i}^{0^{-}}\right\}
$$

Further analysis of $\dot{\sim} 0$ and $\dot{\sim} 1$

If there is $\dot{\sim} 0$ potential $a, \forall 2 \in a$; there is $\dot{\sim} 1$ potential $b, \forall_{\rightarrow} 2 \in b$. We can find $\forall 2 \rightarrow \forall \rightarrow 2$, that is, $2 \neq 2^{*}$, which is changed as $2 \rightarrow 2^{*}$.

According to the property (24) formula, it can be deduced from the potential of natural number set and real number set.

$$
2\left\{\forall 2 \rightarrow \forall_{\rightarrow} 2\right\} \text {, that is } 2\left\{2 \rightarrow 2^{*}\right\} ; \forall 2 \in \dot{\sim} 0, \forall_{\rightarrow} 2 \in \dot{\sim} 1
$$

According to (24) and (25), we can know whether there are two 2 in the potential of natural number set. Whether there are two $2^{*}$, in the potential of real number set. Their relationship: $2\left\{2 \rightarrow 2^{*}\right\}$. There are two 2 potentials in a natural set. They are different. They are called: $2^{\rightarrow} ; 2^{\leftarrow}$. There are two 2 potentials in the real number set. They are different. They are called: $2_{0^{-}}^{*} 2_{0^{+}}^{*}$

$\left\{\left[2^{\rightarrow}, 2^{\leftarrow}\right] \rightarrow\left[2_{0^{-}}^{*}, 2_{0^{+}}^{*}\right]\right\}$ exists, if changed to

$\left\{\left[2^{\rightarrow}, 2_{0^{-}}^{*}\right] \rightarrow\left[2^{\leftarrow}, 2_{0^{+}}^{*}\right]\right\}$ From this to general. Simplification of (24)

$\left\{\left[\forall(\mathrm{U}) \Delta^{\rightarrow} a_{i}^{0^{-}}, \forall(\mathrm{U}) \Delta^{\leftarrow} a_{i}^{0^{-}}\right]\right\} \rightarrow\left\{\left[\forall(\mathrm{U}) \Delta_{0^{-}}^{*} a_{i}^{0^{-}}, \forall(\mathrm{U}) \Delta_{0^{+}}^{*} a_{i}^{0^{-}}\right]\right\}=\left\{\left[\forall(\mathrm{U}) \Delta^{\rightarrow} a_{i}^{0^{-}}, \forall(\mathrm{U}) \Delta_{0^{+}}^{*} a_{i}^{0^{-}}\right]\right\} \rightarrow\left\{\left[\forall(\mathrm{U}) \Delta^{\leftarrow} a_{i}^{0^{-}}, \forall(\mathrm{U}) \Delta_{0^{-}}^{*} a_{i}^{0^{-}}\right]\right\}$

Passing to the limit in (26), we get

$$
\operatorname{Lim}\left\{\left[\forall(\mathrm{U}) \Delta^{\rightarrow} a_{i}^{0^{-}} \rightarrow \forall(\mathrm{U}) \Delta_{0^{+}}^{*} a_{i}^{0^{-}}\right],\left[\forall(\mathrm{U}) \Delta^{\leftarrow} a_{i}^{0^{-}}, \forall(\mathrm{U}) \Delta_{0^{-}}^{*} a_{i}^{0^{-}}\right]\right\}=\forall(\mathrm{U}) \Delta a_{i}^{\uparrow} \wedge \forall(\mathrm{U}) \Delta a_{i}^{\downarrow}
$$

Passing to the limit in the right-hand side of (27), we infer

$$
\operatorname{Lim}\left[\forall(U) \Delta a_{i}^{\uparrow} \wedge \forall(U) \Delta a_{i}^{\downarrow}\right]=\forall \Delta a_{i}^{\uparrow} \wedge \forall \Delta a_{i}^{\downarrow}
$$

In (28), $a_{i}^{\uparrow}$ is the limit potential of mixing, $a_{i}^{\downarrow}$ is the limit potential of mixing. And simplify it.

$$
\begin{gathered}
\forall \Delta a_{i}^{\uparrow} \wedge \forall \Delta a_{i}^{\downarrow}=\forall\left(\Delta a_{i}^{\uparrow} \wedge \Delta a_{i}^{\downarrow}\right) \\
\forall\left(\Delta a_{i}^{\uparrow} \wedge \Delta a_{i}^{\downarrow}\right)=a_{i}^{\uparrow \downarrow}+\operatorname{Lim} \int^{\Delta} 0^{\infty} a_{i+\Delta \varepsilon}^{\uparrow \downarrow}=a_{i}+\operatorname{Lim} \int^{\Delta} 0^{-} a_{i+\Delta \varepsilon}^{\uparrow \downarrow}
\end{gathered}
$$

$\because a_{i}^{\uparrow}, a_{i}^{\downarrow}$ is the limit potential of mixing, $\therefore a_{i}$ is also the potential of mixing; i.e 
$\operatorname{Lim} \int^{\Delta}{ }_{0}^{\infty} a_{i+\Delta \varepsilon}^{\uparrow \downarrow} \in\{N, R\}$, and $N$ is the set of natural numbers, $R$ is the set of real numbers.

$\because \operatorname{Lim} \int_{0^{-}}^{\Delta a_{i+\Delta \varepsilon}^{\uparrow \downarrow}}$ is the infinitesimal infinitesimal; it is the category of the theory of hyperfinity.

$$
a_{i}+\operatorname{Lim} \int^{\Delta} 0^{\infty} a_{i+\Delta \varepsilon}^{\uparrow \downarrow}
$$

On the extension of the meaning of the potential of (30) infinite partition class, it embodies the symmetry relation that the smallest element after infinite partition is 2 .

$$
a_{i}+\operatorname{Lim} \int^{\Delta}{ }_{0}^{\infty} a_{i+\Delta \varepsilon}^{\uparrow \downarrow}, \text { and } a_{i}+\operatorname{Lim} \int^{\Delta}{ }_{0}^{\infty} a_{i+\Delta \varepsilon}^{\uparrow} \text { and } a_{i}+\operatorname{Lim} \int^{\Delta} 0^{\infty} a_{i+\Delta \varepsilon}^{\downarrow}
$$

The meaning of this pattern is far-reaching.

$2\left\{\left[a_{i}+\operatorname{Lim} \int^{\Delta} 0^{\infty} a_{i+\Delta \varepsilon}^{\uparrow}\right] \rightarrow\left[a_{i}+\operatorname{Lim} \int^{\Delta} 0^{\infty} a_{i+\Delta \varepsilon}^{\downarrow}\right]\right\}$

The meaning of relation (32) is the same as that of $2\{1 \rightarrow 1\}$, and the elements with four potentials are simplified from (24) and (25), namely:

$$
2\left\{\forall(\mathrm{U}) \Delta a_{i}^{0^{-}}, \forall(\mathrm{U}) \Delta^{*} a_{i}^{0^{-}}\right\}=2\left\{\forall(\mathrm{U}) \Delta a_{i}^{0^{-}} \rightarrow \forall(\mathrm{U}) \Delta^{*} a_{i}^{0^{-}}\right\}
$$

The above formulas and (32) are all elements of four potentials. So (32) the derivation is correct, take a pair of relations:

$$
\left[a_{i}+\operatorname{Lim} \int^{\Delta} 0^{\infty} a_{i+\Delta \varepsilon}^{\uparrow}\right] \rightarrow\left[a_{i}+\operatorname{Lim} \int^{\Delta} 0^{-} a_{i+\Delta \varepsilon}^{\downarrow}\right]
$$

From this, we can realize symmetry and order, and understand that disorder is also temporary.

\subsection{It Embodies the Dynamic Law of Things and Four Mixed Potentials Belong to the Category of the Theory of Hyperfinity}

$$
\left\{\begin{array}{l}
{\left[a_{i}+\operatorname{Lim} \int^{\Delta} 0^{\infty} b_{i+\Delta \varepsilon}\right] \rightarrow\left[a_{i}+\operatorname{Lim} \int^{\Delta} 0^{+} b_{i+\Delta \varepsilon}\right]} \\
{\left[a_{i}+\operatorname{Lim} \int^{\Delta} 0^{\infty} b_{i+\Delta \varepsilon}^{\uparrow}\right] \rightarrow\left[a_{i}+\operatorname{Lim} \int^{\Delta} 0^{\infty} b_{i+\Delta \varepsilon}^{\downarrow}\right]}
\end{array}\right.
$$

(35) It can be seen that the minimum element after infinite classification is 2 , and four mixed potentials are formed, which belongs to the category of the theory of hyperfinity. Because the minimum element of mixed potential is infinitesimal infinitesimal, there is

$a_{i}+\operatorname{Lim} \int^{\Delta} 0^{\infty} a_{i+\Delta \varepsilon}^{\uparrow \downarrow}$ is always accompanied by $a_{i}$ potential So $a_{i}+\operatorname{Lim} \int^{\Delta} 0^{\infty} a_{i+\Delta \varepsilon}^{\uparrow \downarrow}$ is a discontinuous potential. $\therefore \dot{\sim} 0<x<\dot{\sim} 1$ not established, Proof completed.

\section{Conclusion}

\subsection{There Is No Continuity Between Potential of the Natural Number Set and the Real Number Set}

The smallest element after infinite partition is 2 , which forms four mixed potentials. The smallest element is infinitesimal of infinitesimal, which belongs to the category of transfinite theory. Georg Cantor's conjecture about the continuity of set potential is proved.

\subsection{The Infinite Partition Class and the Continuity Problem of Set Potential Is Constructed by Differential Incremental Equilibrium Theory}

Through the limit potential of differential increment, four mixed potentials with infinitesimal minimum element are formed. That is, $a_{i}+\operatorname{Lim} \int^{\Delta} 0^{-} a_{i+\Delta \varepsilon}^{\uparrow \downarrow}$ is always accompanied by $a_{i}$ potential. So $a_{i}+\operatorname{Lim} \int^{\Delta} 0^{\infty} a_{i+\Delta \varepsilon}^{\uparrow \downarrow}$ is a discontinuous potential. Cantor's conjecture is proved that the potential of the set of natural numbers and the set of real numbers is discontinuous.

\section{References}

[1] Zhang Wenxiu, Qiu Guofang, Uncertain Decision Making Based on Rough Sets, Beijing China, tsinghua university press, 2005: 1-255.

[2] Zheng Weiwei, Complex Variable Function and Integral Transform, Northwest Industrial University Press, 2011: 1-396.

[3] Lou Senyue, Tang Xiaoyan, Nonlinear Mathematical Physics Method, Beijing China, Science Press, 2006: 1-365.

[4] Zhu Rongrong, Differential Incremental Equilibrium Theory, Fudan University, Vol 1, 2007: 1-213.

[5] LABPHTEB M. A., TriaBAT B. B., Methods of Function of a Complex Variable Originally published in Russian under the title, 1956, 2006: 1-287.

[6] Numerical Treatment of Multi-Scale Problems Porceedings of the $13^{\text {th }}$ GAMM-Seminar, Kiel, January $24-26,1997$ Notes on Numerical Fluid Mechanics Volume 70 Edited By WolfGangHackBusch and Gabriel Wittum.

[7] Zhu Rongrong, Differential Incremental Equilibrium Theory, Fudan University, Vol 2, 2008: 1-352.

[8] C. Rogers W. K. Schief, Bäcklund and Darboux Transformations: Geometry and Modern Applications in SolitionTheory, first published by Cambridge University, 2015: 1-292.

[9] Gu chaohao, $\mathrm{Hu}$ Hesheng, Zhou Zixiang, DarBoux Transformation in Solition Theory and Its Geometric Applications (The second edition), Shanghai science and technology Press, 1999, 2005: 1-271.

[10] W. Miller, Symmetry Group and Its Application, Beijing China, Science Press, 1981: 1-486. 
[11] Gong Sheng, Harmonic Analysis on Typical Groups Monographs on pure mathematics and Applied Mathematics Number twelfth, Beijing China, Science Press, 1983: 1-314.

[12] Ren Fuyao, Complex Analytic Dynamic System, Shanghai China, Fudan University Press, 1996: 1-364.

[13] Chen Zhonghu, Lie group guidance, Higher Education Press, 1997: 1-334.

[14] Su Jingcun, Topology of Manifold, Wuhan China, wuhan university press, 2005: 1-708.

[15] Wu Chuanxi, Li Guanghan, Submanifold geometry, Beijing China, Science Press, 2002: 1-217.

[16] Ding Peizhu, Wang Yi, Group and its Express, Higher Education Press, 1999: 1-468.
[17] Zheng jianhua, Meromorphic Functional Dynamics System, Beijing China, tsinghua university press, 2006: 1-413.

[18] Xiao Gang, Fibrosis of Algebraic Surfaces, Shanghai China, Shanghai science and technology Press, 1992: 1-180.

[19] Jari Kaipio Erkki Somersalo, Statistical and Computational Inverse Problems With 102 Figures, Spinger.

[20] E. M. Chirka, Complex Analytic Sets Mathematics and Its Applications, Kluwer Academic Publishers Gerald Karp, Cell and Molecular Biology: Concepts and Experiments (3e), Higher Education Press, 2005: 1-792.

[21] Qiu Chengtong, Sun Licha, Differential Geometry Monographs on pure mathematics and Applied Mathematics Number eighteenth, Beijing China, Science Press, 1988: 1-403. 